\title{
Optimization of a Piezoelectric Crystal Driver Stage using System Simulations
}

\author{
Jonny Johansson \\ Luleå University of Technology \\ 97187 Luleå, Sweden
}

\begin{abstract}
Using SPICE, successful efforts have previously been made in modeling piezoelectric devices and their functionality. In this paper the piezoelectric device is simulated together with MOS transistor models. The design of a CMOS piezoelectric crystal driver stage is presented. Measurements on a manufactured chip verify the chosen design approach and the performance predicted by the simulations. In the work, achieving small silicon area while maintaining maximum possible output ultrasound pulse amplitude has been a key criterion. The driver stage has been implemented using a $0.6 \mu \mathrm{m}$ CMOS process. Measurements and simulations have been performed using PZ-27 crystals without backing. Results clearly show that the performance of a complete system comprising both piezoelectric and electronical devices can be predicted with good accuracy using the proposed SPICE simulation approach.
\end{abstract}

\section{INTRODUCTION}

In the area of ultrasound pulse generation, two main routes are present in the literature. One is dealing with integrating complete ultrasound devices including the active element into very small units [1] - [3]. The other concerns generation of ultrasound pulses without regarding size or power consumption for the pulse generation electronics [4] - [6]. However, the area of miniaturization of pulse electronics for use with standard ultrasound crystals is sparsely exploited. The work in this paper is aimed at creating a design method for driver electronics for ASIC integration, and showing that simulations can be used to predict the performance of a complete system.

The design of a CMOS piezoelectric crystal driver stage is presented. SPICE models for the piezoelectric crystal and the acoustic materials have been used in simulations together with semiconductor models and data from the ASIC manufacturer, allowing optimization of the complete system. Measurements on a manufactured chip verify the chosen design approach and the performance predicted by the simulations.

An important aspect during the design work has been to minimize the consumed silicon area while maintaining maximum possible ultrasonic output signal amplitude. The resulting circuit is intended to be used as a block in a complete mixed mode ASIC for ultrasound transmission, receiving and signal conditioning.

\section{DESIGN}

\section{Design environment}

The design and simulations have been made using the Cadence IC 4.43 design framework. Simulations used the Cadence Spectre simulation engine. The target process for the implementation of the design is a $0.6 \mu \mathrm{m}$ CMOS process.

\section{Ultrasound device}

The SPICE models used in the work are those presented by van Deventer [7]. The ultrasound device is a PZ-27 piezoceramic disc manufactured by Ferroperm, Denmark. The disc has a diameter of 16 $\mathrm{mm}$ and a thickness of $487 \mu \mathrm{m}$, giving a fundamental frequency of $4 \mathrm{MHz}$ corresponding to a period time $T$ of $250 \mathrm{~ns}$. It has been of interest to verify the design approach using different capacitive transducer loads. Thus, simulations and measurements have been carried out for one single disc and for two discs connected in parallel. In both cases, the discs are glued with cyanoacrylate glue to a $15 \mathrm{~mm}$ thick plate of PMMA. No backing material is used for the crystals. The maximum peak-to-peak amplitude for 


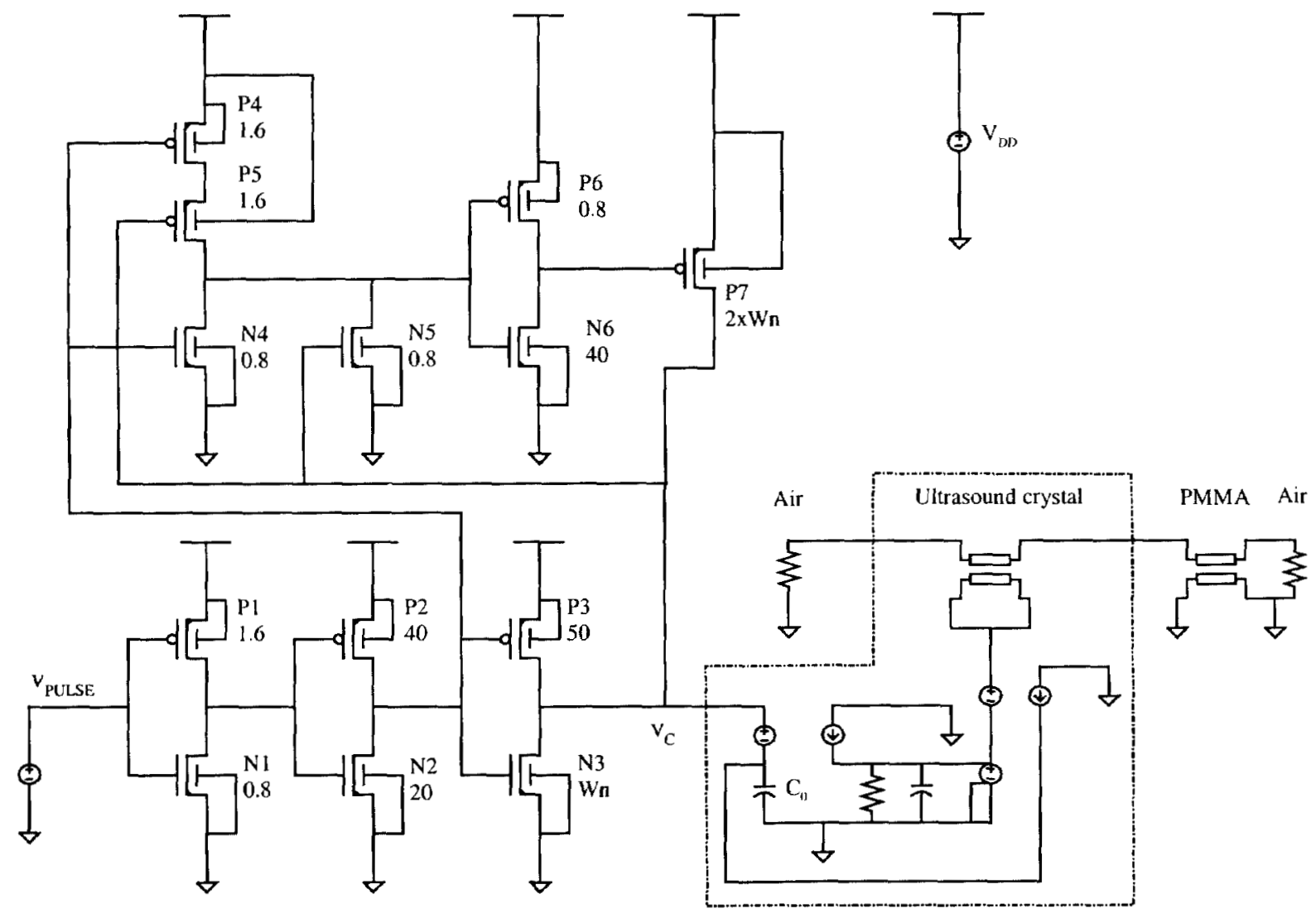

Figure 1: Circuit used for system simulations.

the echo received from the PMMA-air interface has been used as a measure of output pulse amplitude.

According to van Deventer [7], the resistive part in the transmission line model of PMMA, modelling the acoustic attenuation, is frequency dependent. However, this is not modelled, but the parameters are calculated at the resonance frequency of the crystal. Comparisons between measurements and simulations on the PMMA model also clearly show that high frequency components in a transmitted signal are attenuated more in reality than in the simulations. To create a first-order model of this effect, all received signals for the simulations in this work are measured after passing a first order low pass filter with a corner frequency of $400 \mathrm{kHz}$.

\section{Design considerations}

The driver is designed to be integrated into a larger standard CMOS design. Thus, emphasis has been made to avoid external components and to keep the silicon area minimal. Also, in the $0.6 \mu \mathrm{m}$ process used, available power supply is restricted to $+5 \mathrm{~V}$. In achieving maximum output signal amplitude while holding these constraints, the shape of the excitation pulse applied to the crystal is decisive. As the design is focused on pulse amplitude rather than on short pulse time, it was decided to use a single square wave excitation pulse with a pulse time of half the crystal oscillating period time [6].

Also, the fall and rise times of the discharging and charging of the crystal are important in getting maximum amplitude from the ultrasound pulse. The choice of large driving transistors can decrease the times giving a higher output amplitude. On the other hand, this consumes chip area and increases the load on the preceding transistor stage as well as on the crystal. Simulations of the PZ-27 crystal driven by a pulse source with adjustable fall and rise times, show that setting the times to $1 / 10$ of the crystal resonance 
frequency period time gives maximum pulse amplitude from the crystal while avoiding to use faster transitions than necessary.

The driver stage is designed to be controlled by an external pulse source. One pair of push-pull transistors is used as main output transistors to give good control of rise and fall behaviour. In order to achieve good receiving conditions for the returned echo, the output transistors are automatically set to high impedance after the pulse has been sent.

\section{Circuit functionality}

This section gives a qualitative description of the circuit. The complete circuit is shown in figure 1. All transistors are minimum length $(0.6 \mu \mathrm{m})$, with widths as given in the figure.

There are two main driving transistors, N3 for discharging and P7 for charging. The starting state for the crystal is charged, being held by P3. N3 is controlled directly by the input via two inverters, while P7 is controlled by the input signal AND gated with the output crystal voltage. Thus, for P7 to be able to conduct, both the input and the output should be low. One pulse operation is described below:

1. The US crystal is held at $V_{D D}$ via the $\mathrm{P}$ channel transistor P3. P7 and N3 are blocked.

2. When a positive pulse is applied to the input, the crystal is rapidly discharged via transistor N3. P7 is still blocked.

3. As the input voltage returns to ground, N3 blocks and $\mathrm{P} 3$ again starts to slowly charge the crystal. Also, P7 starts to conduct and rapidly charges the crystal up to $V_{D D^{*}}$

4. As Vc again reaches $V_{D D}, \mathrm{P} 7$ is blocked and the pulse cycle is completed.

\section{Transistor dimensioning}

As discussed above, the driving transistors N3 and P7 should be dimensioned to achieve rise and fall times less than $T / 10$, where $T$ is the period time of the crystal resonance frequency. To get a starting point for the dimensioning, an approximate formula for charging time was used. This was derived from approximating the transistor characteristic at maximum gate voltage with two straight lines as shown in figure 2.

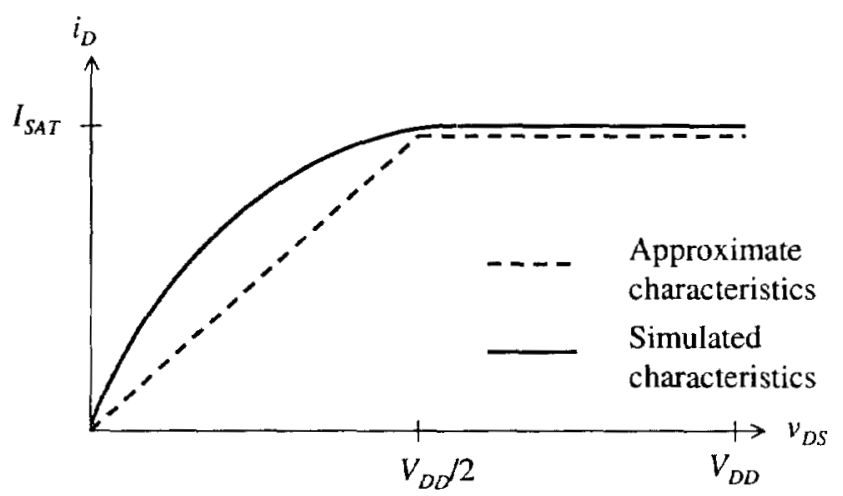

Figure 2: Approximation of transistor characteristics.

The static crystal capacitance $\mathrm{C}_{0}$ is then discharged to $5 \%$ of $V_{D D}$ within the time

$$
t_{f}=1.65 \cdot \frac{C_{0} \cdot V_{D D}}{I_{S A T}},
$$

where $I_{\mathrm{s} s r}$ is the saturation current for the transistor used. For given $t_{f}$ and $C_{p}$, the approximation gives a conservative value of $I_{S A T}$. This is useful as it helps to account for process variations. Using the given $I_{S A} / \mu \mathrm{m}$ for the chosen CMOS process, the required transistor widths for a single $16 \mathrm{~mm}$ PZ27 crystal are calculated to $3.4 \mathrm{~mm}$ for $\mathrm{N} 3$ and $6.8 \mathrm{~mm}$ for P7. For two parallel crystals $C_{0}$ is doubled giving the widths of $6.8 \mathrm{~mm}$ for $\mathrm{N} 3$ and $13.6 \mathrm{~mm}$ for P7.

The input impedance of the driver stage when receiving an echo signal from the crystal is decided by transistor P3. Thus, the width of P3 is chosen to give dynamic impedance in the conducting state that is larger than the crystal impedance at the resonance frequency. Transistors P2, N2 and N6 are dimensioned to be able to drive the gates of the driving transistors $\mathrm{N} 3$ and $\mathrm{P} 7$ with at least a rise/fall time of $T / 10$. Here, the resulting minimum values were multiplied by a factor of 5 to improve the balance in the inverter chain and reduce switching losses for lower loads than the target crystal [8].

The size of transistor P6 is made small in order to prolong the turn off time for $\mathrm{P7}$ so that the charging of $C_{0}$ will be fully completed before $\mathrm{P7}$ is turned off. The inverter $\mathrm{P} 1 / \mathrm{N} 1$ and the NAND gate $\mathrm{P} 4 / \mathrm{P} 5 / \mathrm{N} 4 / \mathrm{N} 5$ are dimensioned with minimum size transistors, having the $\mathrm{P}$ channel devices 2 times the $\mathrm{N}$ channel devices to achieve balanced performance. 


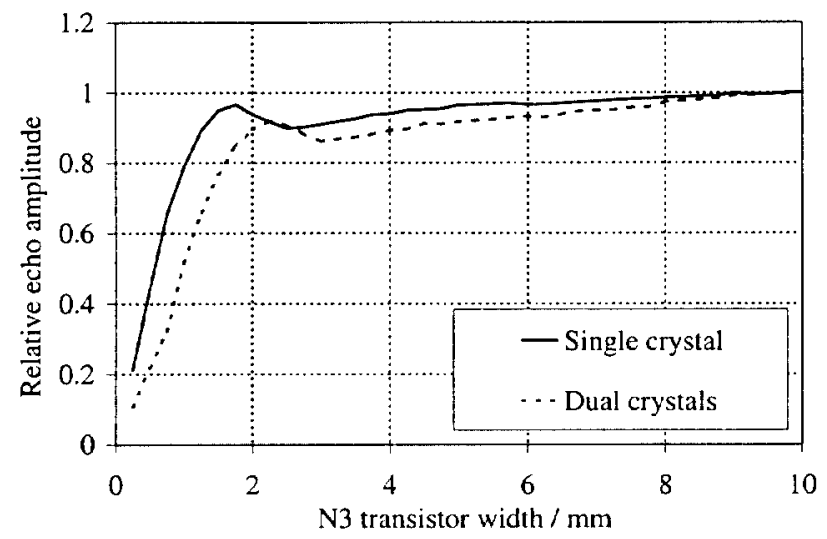

Figure 3: Simulation using ideal components showing relative echo amplitude vs. transistor width.

\section{Simulations}

\section{Ideal driver model}

As a starting point, simulations were made on one driver stage where the widths of the driving transistors $\mathrm{N} 3$ and $\mathrm{P} 7$ were varied. The ratio $\mathrm{P} 7 / \mathrm{N} 3$ was kept constant at 2 to keep balanced performance. Simulations were made using typical transistor performance for the chosen process, and no internal or external parasitics were included at this time. Results for single and dual crystals are shown in figures 3 and 4 . The amplitudes on the $y$-axis are normalized at $\mathrm{N} 3=10 \mathrm{~mm}$ for the curves individually.

To verify the simulations, it was desirable to measure on a range of transistor widths as shown in figure 3. Thus, it was decided to implement the driver stages with three different sizes $(2 \mathrm{~mm} / 4 \mathrm{~mm}$, $1 \mathrm{~mm} / 2 \mathrm{~mm}$ and $0.5 \mathrm{~mm} / 1 \mathrm{~mm}$ ) for the output transistors N3/P7. Each manufactured die contains 2 each of the above mentioned sizes. In order to achieve larger widths for measurements several stages and dies are connected in parallel.

\section{Simulating the manufactured chip}

Simulations were also made using a model for the driver stage including parasitic capacitance extracted from the actual chip layout. Also package parasitics and external wiring parasitics were included.

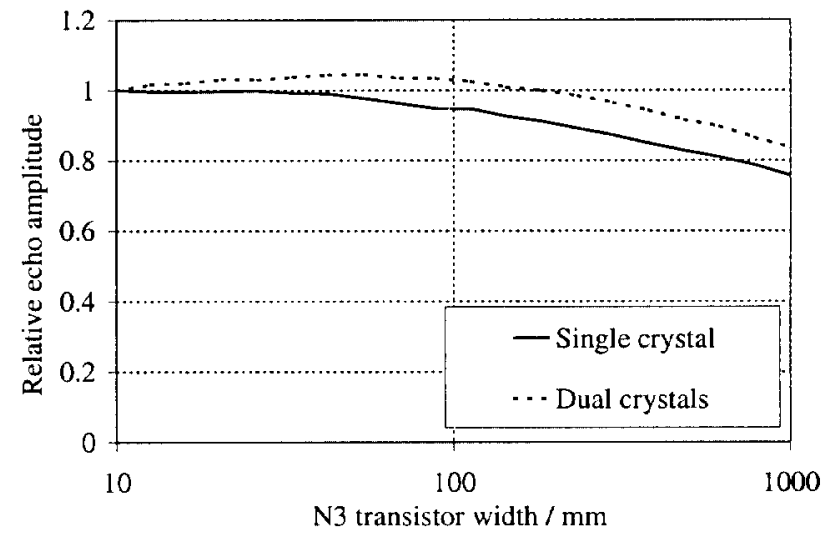

Figure 4: Simulation using ideal components showing relative echo amplitude vs. transistor width.

In order to achieve different output transistor widths different number of drivers were connected in parallel as also done during measurements. Results from the simulations are shown in figure 5 for the single crystal and in figure 6 for the dual crystals. Due to changes in the loading of the crystal when several stages are connected in parallel, the resulting curve has been divided into sections dependant on the number of transistor stages used. Loading on the crystal when receiving an echo stems from the pull up transistor P3 and the different parasitics mentioned above.

\section{MEASUREMENTS}

Measurements were made on the fabricated chip for a total output transistor width up to $9 \mathrm{~mm}$ for N3, corresponding to $18 \mathrm{~mm}$ for P7. Different number of driver stages were used in parallel to achieve different total output transistor widths. Results from the measurements are presented in figure 7 for the single crystal and in figure 8 for the dual crystal. The curves have been divided into sections dependant on the number of driver stages used as discussed for the simulations above. 


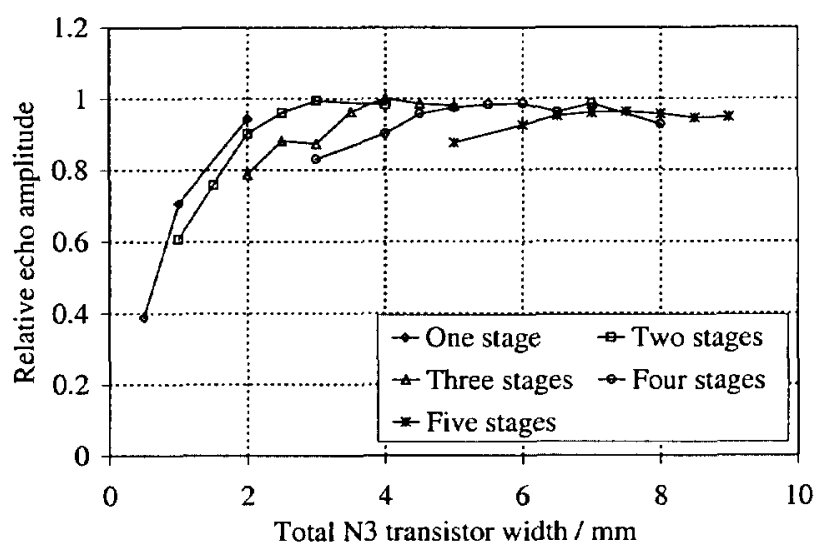

Figure 5: Simulation on a single crystal using a non-ideal electronic model showing normalized echo amplitude as function of transistor width.

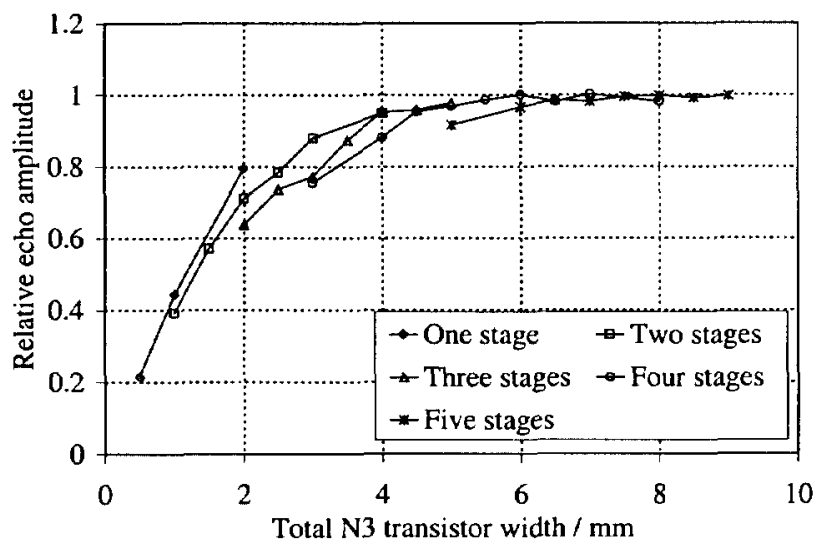

Figure 6: Simulation on dual crystals using a nonideal electronic model showing normalized echo amplitude as function of transistor width.

\section{DISCUSSION}

\section{Simulations}

The results from the simulations with ideal electronic models (fig. 3) show a peak in echo amplitude around the $2 \mathrm{~mm}$ transistor width for both the single and dual crystals, after which a slight drop takes place. One possible explanation to this behaviour is that the $Q$ of the LCR parallel oscillation circuit formed by the crystal and N3 is dependent on the transistor width. For small transistor width the dynamic impedance in conducting state for the transistor is high leading to a high $\mathrm{Q}$ during the excitation pulse. As the width increases the $Q$ value decreases and settles to a value decided by the crystal. The peak is not seen in the simulations where

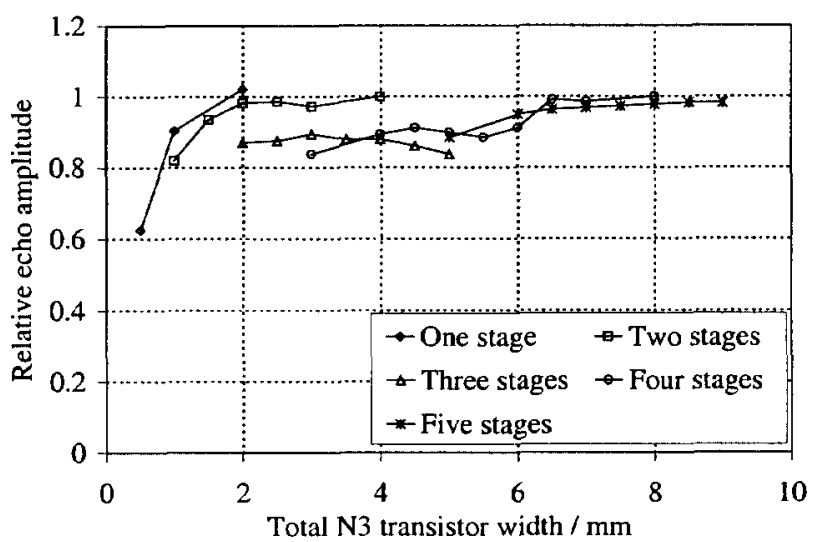

Figure 7: Measurement on a single crystal showing normalized echo amplitude as function of transistor width.

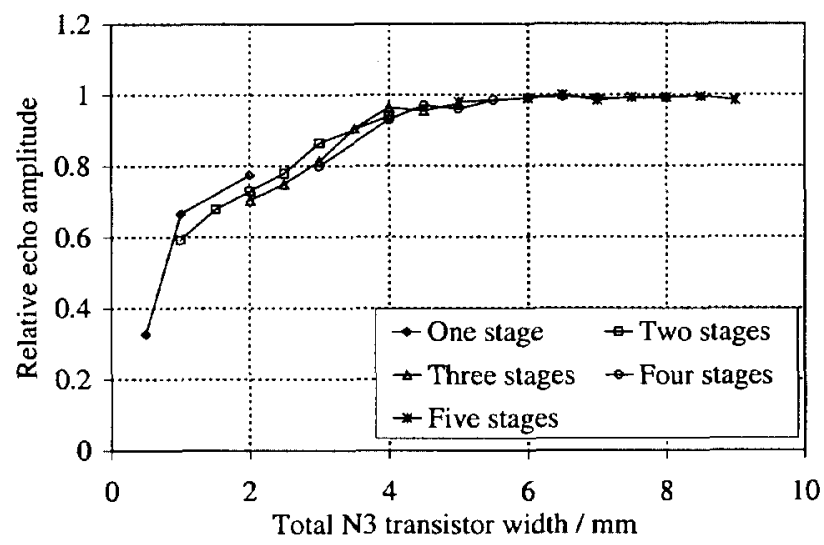

Figure 8: Measurement on dual crystals showing normalized echo amplitude as function of transistor width.

parasitic effects are taken into account (fig. 5, 6). This is likely due to the parasitic L, R and C lowering the $\mathrm{Q}$ of the circuit from the start. In these simulations a slight drop in echo amplitude can be observed as more driver stages are connected. The maximum output amplitude stabilises as the width passes $3 \mathrm{~mm}$ for the single crystal (fig. 5) and $6 \mathrm{~mm}$ for the dual crystal (fig. 6).

The ideal simulations (fig. 3,4) show a gradual increase in echo amplitude from $3 \mathrm{~mm}$ transistor width up to $10 \mathrm{~mm}$ for the single crystal and $50 \mathrm{~mm}$ for the dual crystal. After these points the echoes decrease (fig. 4). This is due to the fact that the load capacitance presented by the drains of $\mathrm{N} 3$ and $\mathrm{P7}$ is increasing. In the simulations where parasitics are 
included (fig. 5, 6), the gradual increase in the range 3 to $10 \mathrm{~mm}$ can not be seen. Probable cause for this difference is that the load capacitance in these cases are dependent also on pads and the amount of metal used in the chip, giving a higher value than for the drain on the transistors only.

\section{Measurements}

The measurements for the single crystal (fig. 7) show a higher dependence on the number of stages used than what is seen in the simulations. Probably this is due to the parasitics not being correctly estimated in the simulations. However, measurements and simulations show a fair agreement in the dependence on transistor width if the load influence is excluded.

For the dual crystal the measurements presented in figure 8 show that the load influence is smaller, which is to be expected as the source impedance for the parallel crystals is half of the source impedance for a single crystal. For the dual crystal, measurements and simulations agree well in the dependence on transistor width.

\section{Design}

Results from both simulations and measurements suggest that the calculated widths give more than $90 \%$ of maximum possible output amplitude for a $5 \mathrm{~V}$ push-pull configuration, providing the number of driver stages used is kept minimal. Also, using the calculated widths, a margin of $15-20 \%$ is provided before a drop in output amplitude takes place. This will account for process variations in the manufacturing of the chip.

For the single crystal, using two $2 \mathrm{~mm}$ driver stages gives a transistor area smaller than $0.1 \mathrm{~mm}^{2}$, excluding pads and metal interconnections.

\section{CONCLUSIONS}

System simulations using SPICE models for the piezoelectric crystal together with MOS transistor models have been used with very promising results, in the design of a CMOS driver circuit for piezoelectric crystals. The circuit has been manufactured using a $0.6 \mu \mathrm{m}$ CMOS process. Measurements on the manufactured chip verify the chosen design strategy and the performance predicted by the simulations. The results show that simulations can be used to optimize the performance of a complete system comprising both piezoelectric and electronical devices. This indicates that a purely simulation based design strategy can be proposed based on the findings.

\section{REFERENCES}

[1] J.V. Hatfield et al, "An integrated multielement array transducer for ultrasound imaging", Sensors and Actuators A, 41-42, pp. 167-173, 1994.

[2] W.C. Black and D.N. Stephens, "CMOS Chip for Invasive Ultrasound Imaging", IEEE Journal of Solid State Circuits, Vol. 29, No. 11, pp. 1381-1387, November 1994.

[3] C Kuratli and Q Huang, "A Fully Integrated Self-Calibrating Transmitter/Receiver IC for an Ultrasound Presence Detector Microsystem", IEEE Journal of Solid State Circuits, Vol. 33, pp. 832-841, No. 6, June 1998.

[4] R.Y. Liu, "The design of electric excitations for the formation of desired temporal responses of highly efficient transducers", Acoustical Imaging, Vol. 12, pp. 293-305, 1982.

[5] S.S. Muhlen, "Design of an optimized highpower ultrasonic transducer", IEEE 1990 Ultrasonics Symposium Proceedings, 3 vol. pp. 1631-1634, 1990.

[6] H.W. Persson, "Electric excitation of ultrasound transducers for short pulse generation", Ultrasound in Med. \& Biol., Vol. 7, pp. 285291, 1981.

[7] J.A. van Deventer et al, " PSpice simulation of ultrasonic systems", IEEE Transactions on Ultrasonics, Ferroelectrics and Frequency Control, Vol. 47, No. 4, pp. 1014-1024, July 2000.

[8] J. M. Rabaey, Digital Integrated Circuits, New Jersey: Prentice Hall, 1996, ch. 4, pp. 243. 